\title{
Neurologic Presentation of Kawasaki Disease
}

\section{Noopur Goyal ${ }^{1} \cdot$ Debasree Gangopadhyay ${ }^{1} \cdot$ Manisha Chakrabarti ${ }^{2} \cdot$ Mahua Roy $^{1} \cdot$ Biswajit Bandyopadhyay $^{1}$}

Received: 30 April 2019 / Accepted: 7 April 2020 / Published online: 17 April 2020

(C) Dr. K C Chaudhuri Foundation 2020

To the Editor: Kawasaki Disease (KD) is a multisystem vasculitis with known neurologic involvement, but central nervous system (CNS) complications as initial presentation of $\mathrm{KD}$ are extremely rare [1]. We present two cases of KD with neurologic presentation.

A three-year-old-girl, with history of high grade fever, rash and redness of eyes, developed extreme irritability and ataxia, on day 3. Cerebrospinal fluid (CSF) examination, nerve conduction study and magnetic resonance imaging of brain were normal. Laboratory studies showed high normal leukocyte and platelet count, high erythrocyte sedimentation rate (ESR) and C-reactive protein (CRP). Serum electrolytes, renal and hepatic function were normal, with negative serology for dengue, scrub typhus and leptospirosis and negative work up for immune mediated ataxia. On day 6, patient developed right hemiparesis with persistently high ESR, CRP, leukocyte and platelet count and a normal echocardiogram. With working diagnosis of KD, intravenous immunoglobulin (IVIG) ( $2 \mathrm{~g} / \mathrm{kg}$ over $12 \mathrm{~h}$ ) was given with aspirin $(80 \mathrm{mg} / \mathrm{kg} / \mathrm{d}$ for $3 \mathrm{~d}$, followed by $5 \mathrm{mg} / \mathrm{kg} /$ d for $8 \mathrm{wk}$ ). Patient recovered neurologically with defervescence of fever within $72 \mathrm{~h}$ of treatment. Development of periungual peeling on day 13 of illness along with rising platelet count supported the diagnosis of incomplete KD.

A five-month-old infant, presented with history of fever for $3 \mathrm{~d}$, rash all over body, vomiting and altered sensorium. Patient had tachycardia, hypotension, maculopapular rash, hepatomegaly, depressed sensorium and depressed deep tendon reflexes, on examination. CSF examination was consistent with aseptic meningitis which was treated accordingly. Patient responded to treatment but with recurrence of fever

Noopur Goyal

ar.noopur@gmail.com

1 Department of Pediatric Cardiology, Rabindra Nath Tagore International Institute of Cardiac Sciences, E M Bypass, Kolkata, West Bengal 700099, India

2 Department of Pediatric Cardiology, Indraprastha Apollo Hospitals, New Delhi, India after $5 \mathrm{~d}$ of defervescence. ESR, CRP and platelet count were high. Echocardiogram showed aneurysms of right coronary artery $[4.5 \mathrm{~mm}(\mathrm{z}$ score +9.7$)]$ and left anterior descending artery $[5 \mathrm{~mm}(\mathrm{z}$ score +12.6$)]$. Patient was diagnosed as infection triggered KD and given IVIG (2 g/kg over $12 \mathrm{~h}$ ), aspirin $(80 \mathrm{mg} / \mathrm{kg} / \mathrm{d}$ till $48 \mathrm{~h}$ of defervescence, and continued on $5 \mathrm{mg} / \mathrm{kg} / \mathrm{d}$ ), and warfarin. Patient's fever subsided with no recrudescence and he is presently under follow up for coronary artery aneurysms.

Neurologic involvement in $\mathrm{KD}$ is reported in $1.1 \%$ to $3.7 \%$ of KD cases [2]. It ranges from soft neurologic symptoms like irritability and lethargy to CNS involvement in form of seizures, aseptic meningitis, facial nerve palsy, hemiplegia, cerebral infarction and coma. Role of single-photon emission computed tomography (SPECT) is emerging in evaluation of cerebral hypoperfusion in $\mathrm{KD}$ secondary to cerebral vasculitis [3]. Further studies are needed to assess the incidence of neurologic complications of $\mathrm{KD}$, long term sequelae, role of radiologic imaging, and IVIG in treating these manifestations.

\section{Compliance with Ethical Standards}

Conflict of Interest None.

\section{References}

1. Dhawan SR, Vaidya PC, Suri D, Singh S, Singhi PD. Infantile Kawasaki disease presenting as acute meningoencephalitis. Int $\mathrm{J}$ Rheum Dis. 2017;20:2225-6.

2. Jindal AK, Pilania RK, Suri D, Gupta PC, Singh S. An adult with fever and facial nerve palsy: an unusual presentation of Kawasaki disease. J Clin Rheumatol. 2019. https://doi.org/10.1097/RHU. 0000000000001024.

3. Sato T, Ushiroda Y, Oyama T, Nakatomi A, Motomura H, Moriuchi H. Kawasaki disease-associated MERS: pathological insights from SPECT findings. Brain Dev. 2012;34:605-8.

Publisher's Note Springer Nature remains neutral with regard to jurisdictional claims in published maps and institutional affiliations. 